\title{
$\mu$ Graph: Haptic Exploration and Editing of 3D Chemical Diagrams
}

\author{
Cristian Bernareggi \\ Università degli Studi di Milano \\ Dipartimento di Informatica \\ cristian.bernareggi@unimi.it
}

\author{
Dragan Ahmetovic \\ Università degli Studi di Torino \\ Dipartimento di Matematica \\ dragan.ahmetovic@unito.it
}

\author{
Sergio Mascetti \\ Università degli Studi di Milano \\ Dipartimento di Informatica \\ sergio.mascetti@unimi.it
}

\begin{abstract}
People with visual impairments or blindness (VIB) encounter difficulties in exploring graphical representations that are widely used for the study of STEM subjects. In particular, graphs are used to represent many different scientific notations: flowcharts, automata, cognitive maps, and more. Among these, structural chemical formulae are characterized by a complex, often 3-dimensional structure, which makes them hard to access and author with traditional assistive tools.

We propose $\mu$ Graph, a multimodal system that combines haptic and speech feedback to enable people with VIB to explore and edit structural chemical formulae. Two main contributions are presented: (i) a novel, non-visual interaction paradigm for exploring graphs and its implementation in the $\mu$ Graph system, and (ii) an extensive evaluation of the proposed system with 10 participants with VIB showing that $\mu$ Graph is thoroughly accessible and that the haptic feedback enhances understanding of the geometric properties of a graph.
\end{abstract}

\section{Author Keywords}

STEM education; visual impairment; graph accessibility;

\section{CCS Concepts}

-Social and professional topics $\rightarrow$ Assistive technologies; -Human-centered computing $\rightarrow$ Haptic devices;

\section{INTRODUCTION}

Constructing mental images of complex spatial structures can be difficult for people with visual impairments or blindness (VIB) [10]. Such problem is crucial in STEM education, where mathematical notation [1] and visual representations [19] are commonly used. In particular, graph structures are often used to represent scientific formalisms such as automata, flowcharts or cognitive maps. In chemistry, structural chemical formulae are graph structures used convey complete geometric representations of molecular structures, including atom arrangement and chemical bond properties, many of which cannot be translated to chemical formulae.

\footnotetext{
Permission to make digital or hard copies of all or part of this work for personal or classroom use is granted without fee provided that copies are not made or distributed for profit or commercial advantage and that copies bear this notice and the full citation on the first page. Copyrights for components of this work owned by others than the author(s) must be honored. Abstracting with credit is permitted. To copy otherwise, or republish, to post on servers or to redistribute to lists, requires prior specific permission and/or a fee. Request permissions from permissions@ acm.org.

ASSETS '19, October 28-30, 2019, Pittsburgh, PA, USA

(C) 2019 Copyright held by the owner/author(s). Publication rights licensed to ACM ISBN 978-1-4503-6676-2/19/10 . \$ \$15.00
}

DOI: http: //dx . doi .org/10.1145/3308561. 3353811
Force feedback devices, developed to simulate human haptic perception in a three-dimensional work environment, are used for 3D-modeling, industrial design, telemedicine, and more. By combining haptic interaction with sound and speech feedback, novel assistive tools can be developed to support people with VIB in accessing and manipulating three-dimensional graph representations. Nonetheless, a consolidated interaction paradigm combining audio and haptic feedback to support people with VIB has not emerged yet, in particular for the joint problem of exploring and editing graphs. Prior work explore haptic feedback to teach chemistry to people without VIB [7, $15]$, and there are works on haptic systems for people with VIB that support the exploration of function diagrams, charts, geometric shapes $[4,13,21]$, but not $3 \mathrm{D}$ chemical structures.

To address these issues we present $\mu$ Graph (Multimodal Graph) a system using haptic and speech feedback to enable people with VIB to explore, and also edit 3D graphs. The system is based on a preliminary work [5], extended along three directions: 1) the haptic interaction paradigm is enhanced for graph exploration and editing, 2) a fully functional system prototype is implemented, and 3) extensive usability evaluation of the prototype is conducted with people with VIB, including a comparison with an existing solution without haptic feedback. $\mu \mathrm{Graph}$ has been evaluated for 3D structural chemical formulae graphs exploration and editing, but it can be generalized to other graph structures composed of node and edge elements. Results show that $\mu$ Graph is usable by people with VIB for creating, editing and exploring graphs. Moreover, haptic feedback is shown to improve the understanding of the geometric properties of graphs with respect to a solution without it.

\section{RELATED WORK}

Among the assistive tools to support people with VIB in accessing graphical information, embossed images are effective in conveying two-dimensional content. However, they have limited size and resolution, and need to be specifically created to obtain effective tactile exploration [18]. Audio-tactile representations augment the level of detail of tactile images with audio feedback [9, 8]. However, these are also limited to page size, cannot be edited and only enable access to bidimensional data. 3D printed shapes $[11,6]$ provide accurate $3 \mathrm{D}$ visualization of spatial models, and have even been used for representing molecule structures [16] but they also cannot be modified and require specialized hardware. Modelling sets such as Molymod [14] can be used to create various shapes with no additional hardware, but require a sighted supervisor. 
Among multimodal interactive systems designed to provide access to generic graphical representations, many employ audio and speech interaction [12]. In particular, approaches using sonification and free-form exploration of a touch screen surfaces are suitable for 2D function graph exploration [2]. However, free form exploration is difficult for studying structural chemical formulae, which may present multiple edges from each node and cyclical paths.

Among the solutions that provide access to graphical representations with an underlying graph structure, Balik et al. propose an approach for exploring and editing graphs based on screen reader and keyboard. The solution is effective for understanding connections between nodes, but it does not provide information about geometric properties of the graph [3]. Sorge et al. propose a web system for exploring structural chemical formulae of molecules [20], automatically recognized from SVG files. This solution is accessible via browser and interaction is achieved through keyboard and screen reader. This solution provides different exploration mechanisms and it is very useful for people with sight impairment to access formulae available as SVG images in various sources. Nonetheless, this tool does not provide techniques for editing formulae.

Among the approaches that involve haptic interaction, solutions have been proposed for molecule structure visualizations for sighted users [7, 15], but not for people with VIB. For people with VIB, haptic systems were devised to support the learning of handwriting [4] or to access line shapes and line function graphs $[13,21]$. However, such interaction paradigms do not provide primitives to represent nodes, uni- and bi- directed edges, and labels, which are needed to represent structural chemical formulae. Additionally, prior approaches enable access, but not authoring capabilities for people with VIB, which are vital in educational settings for completing assignments and verifying the acquisition of knowledge.

\section{THE PROPOSED SOLUTION}

The design of the system involved four blind participants including a blind interaction designer expert in accessibility problems and haptic interaction. Four major design challenges were faced in the system design: the choice of the haptic device, the multimodal representation of graphs, the interaction paradigm for creating and editing graphs and the operations for exploring graphs.

\section{Choice of the Haptic Device}

A number of different devices have been proposed for haptic and force feedback interaction, with different working volume sizes and degrees of freedom (DoF), that is directions in which independent motion can occur. For example haptic gloves have per-finger interaction, which requires at least 20 degrees of freedom in order to provide independent manipulation for each finger [22]. Such devices are frequently cumbersome and expensive, but provide a higher expressiveness than devices with a low DoF number. In order to enable 3D manipulation, a device with at least $3 \mathrm{DoF}$ is required. Furthermore, the working volume should be sufficiently ample, and the force feedback should be sufficiently intense to unambiguously perceive distinct graph components (i.e., nodes and edges).

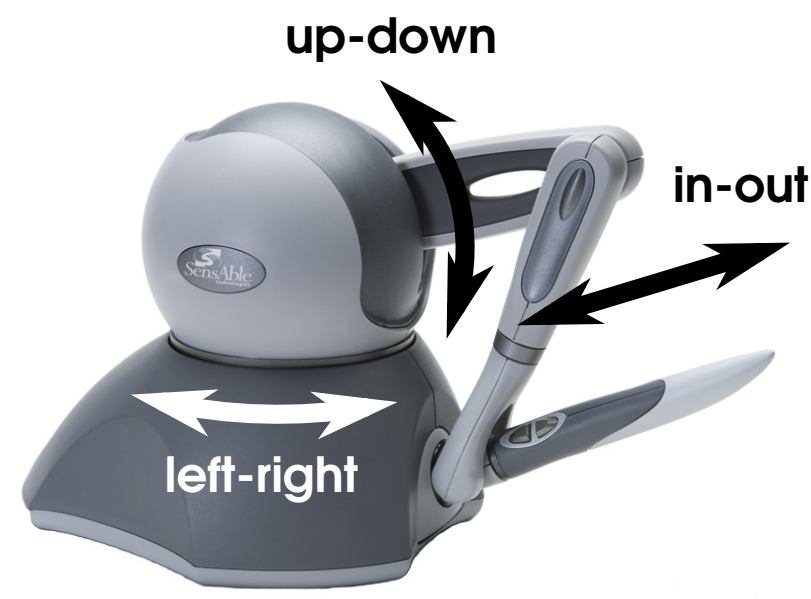

Figure 1: Phantom Omni device by Sensable ${ }^{1}$

The blind interaction designer proposed three candidate haptic devices which have at least $3 \mathrm{DoF}$, different work volumes and force intensity: Sensable Phantom Desktop, Sensable Phantom Omni and Novint Falcon. Three blind subjects graduated in computer science and without any background in haptic interaction were asked to perform three tasks and provide qualitative feedback: recognize an haptic sphere, follow a grid of haptic lines and follow a grid consisting of spheres connected by cylinders. Based on the qualitative feedback provided by the blind subjects, one device that better satisfies their needs, on which detailed results on the kinematic and manipulability have been assessed in prior literature [17] is SensAble Phantom Omni (see Figure $1^{1}$ ). This device consists of a stylus that provides force-feedback in a 3D exploration area of size $16 \times 12 \times 7 \mathrm{~cm}$. Moreover it presents two-button interaction on the stylus (hereby A and B buttons). In addition to the conducted qualitative analysis, Phantom Omni has been previously assessed for shape exploration [5] in terms of $3 \mathrm{D}$ working volume size, force feedback intensity and accuracy. Thus, we select this device for addressing the specified problem.

\section{Graph Representation}

To provide all primitives required for accessing and editing structural chemical formulae, we represent graphs as 3D models of labeled nodes and uni- and bi-directed edges. The nonvisual representation of graphs adopts both haptic feedback and speech messages. Nodes are represented as haptic points that constrain the stylus in the middle of the point through a centripetal force that linearly decreases as the stylus gets far from the point and is null after a given distance. Edges are represented as haptic lines that let the stylus slide along the line. If the user tries to get far from the line, a force directed towards the line brings back the stylus. Information about edge direction and node/edge names is provided through speech feedback.

\footnotetext{
${ }^{1}$ Image retrieved from:

https://www. delfthapticslab.nl/device/phantom-omni/
} 


\section{Interaction Paradigm}

Based on preliminary results [5], four exploration modalities are designed, using the haptic device and a computer keyboard:

- Searching nodes by name: the user types a node name and the haptic device moves the stylus to the node position.

- Free exploration: the user moves the stylus freely and can feel nodes and edges whose names are read.

- Guided nodes exploration: the stylus is moved to each node in the graph automatically. The stylus is held on each node for $1 \mathrm{~s}$ and then moved to the next one. Nodes are ordered based on the distance from the starting exploration point.

- Guided edges exploration: the stylus guides the user in a breadth-first edge exploration. Starting from the node closest to the current position, the stylus automatically moves along all edges connected to that node. For each edge, the stylus moves to the other node and back. When all edges connected to the first node have been explored, the exploration continues with the nearest unexplored connected node. This is iterated until all nodes are explored. The iteration can be stopped by pressing any stylus button.

We describe an example of how these exploration modalities can be used to explore a structural chemical formula. We consider the ammonia molecule shown in the $\mu$ Graph interface in Figure 2. The user activates the guided exploration of nodes in order to find the position and the name of all the atoms in the molecule. Once completed, the user has a mental image of the position of the atoms in the 3D space and their names. Hence, the user can move the stylus freely in the space in order to find a given atom, for example the atom of nitrogen named $\mathrm{N}$. If the user cannot find the atom straightforwardly, it can be searched by name so that the stylus is moved directly to the nitrogen atom. Subsequently, the user is interested to understand the connections in the graph, namely the bonds in the molecule. The user can activate the guided exploration of edges. The stylus is automatically moved along the edge between the nitrogen atom and the first hydrogen atom. Then it is moved back to the nitrogen atom. Again, the stylus is automatically moved from the nitrogen atom to the second hydrogen atom and back. In the end the third bond between nitrogen and hydrogen is explored. At the end of this exploration, the user is aware of the connections between the nitrogen and the hydrogen atoms. In order to check if further connections are present between hydrogen atoms, the user can iterate the guided exploration of edges starting from the hydrogen atoms.

\section{Editing Operations}

$\mu$ Graph supports two editing operations: creation and deletion, for both nodes and edges. The creation of a node is achieved in two steps: first the user selects the node's position by moving the stylus at the desired coordinates and by pressing a stylus button A twice. Audio feedback will read the coordinates of the selected position. Then, the node is inserted by pressing the same button once. Edges are created by selecting the source and the target node through the stylus button B. Deletion of nodes and edges is obtained by positioning the stylus on the target element and double pressing any stylus button. The element is deleted when the user confirms the operation.

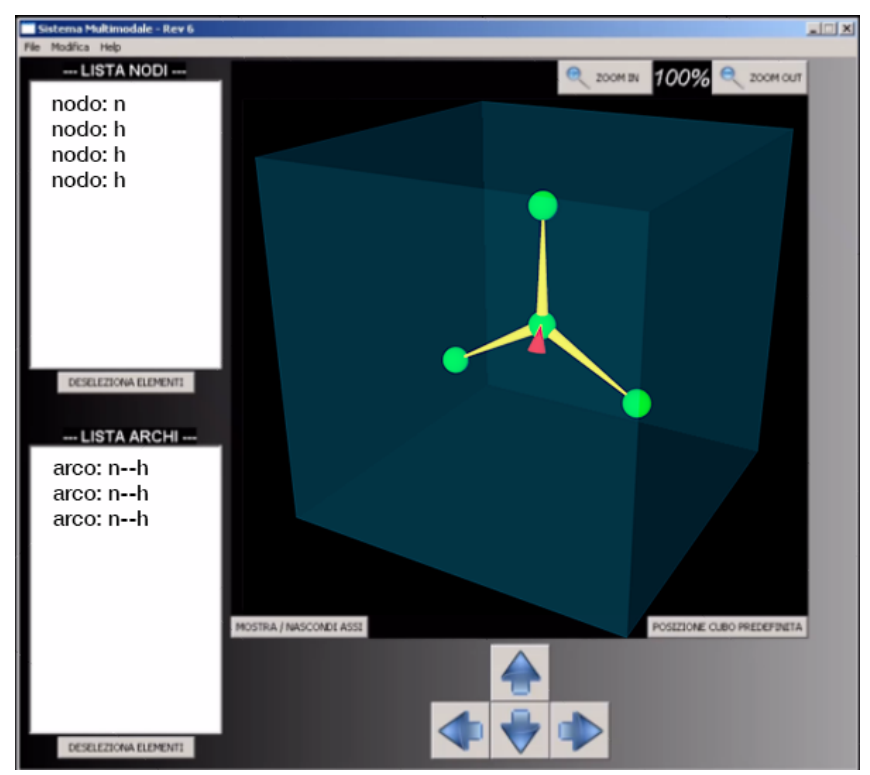

Figure 2: $\mu$ Graph interface showing an ammonia molecule

\section{EVALUATION}

A usability evaluation of $\mu$ Graph for exploration and editing of 3D molecular structure models was conducted with ten blind participants ( 7 males and 3 female aged between 18 and 40 years) in a controlled environment. All the participants were legally blind, all of them knew what is a structural chemical formula and two of them knew many chemical concepts (e.g., oxidation, reduction, the structure of organic molecules as hydrocarbons).

The evaluation had three objectives: 1) to assess the usability of the graph exploration paradigm, 2) to assess the usability of editing operations on graphs, and 3 ) to compare $\mu$ Graph with Accessible Chemical Diagrams (ACD) [20], a prior solution that allows the user to use the keyboard to explore chemical formulae receiving speech feedback only.

The first part of the evaluation consisted in training participants to use $\mu \mathrm{Graph}$ and ACD. Since none of the participants were acquainted with haptic feedback, the training session included three stages: in the first stage (about 15 minutes) the instructor guided the participant to get familiar with the haptic device, in the second stage (about 20 minutes) the participant was instructed on all the system features and in the last stage (about 10 minutes) the participant was free to practice by editing and exploring molecular structures.

The training on ACD was facilitated by the experience of the participants in using screen readers, keyboard and mainstream browsers. The instructors presented the features of ACD in about ten minutes and let the participants practice in exploring a molecular structure for ten additional minutes. After the training session, the participants were required to complete three tasks. At the end of the tasks, the participants were asked to answer open-ended questions about the usefulness of available operations and difficulty of tasks. 


\section{First Task: Exploration}

In the first task the participant is required to explore the structural chemical formula of butane (see Figure 3a) with $\mu$ Graph. The participant is free to use all the exploration modalities. At the end of the exploration the participant is asked to verbally describe the chemical formula to the supervisor. In particular, the participant is requested to report: the name and the number of atoms, the bonds between atoms, the position of atoms in the 3D space by saying their coordinates or by describing their relative positions (e.g., on the same line on the right of the atom of carbon) and the angles between edges.

An atom position is considered correct if described within $2 \mathrm{~cm}$ from the intended position, while a bond is considered correct if within $15^{\circ}$ from the correct direction. These thresholds are defined, considering the dimension of the workspace, so that it is still possible to disambiguate between nearby atoms and directions, even if an error of such entity is committed.

\section{Second Task: Editing}

In the second task the participant is required to create a structural chemical formula of the ethane (see Figure $3 b$ ) in $\mu$ Graph. The molecular structure is first verbally described, and repeated upon request, by informing the participant about the coordinates of the position of atoms, the name of atoms and the connections between atoms. The participant is free to use all the editing operations. Furthermore, the participant can use reading of the coordinates and exploration operations to check the inserted data. Correctness thresholds for this task are the same as in the previous one $\left(2 \mathrm{~cm}\right.$ for atom positions, and $15^{\circ}$ for bond directions).

\section{Third Task: Comparison with ACD}

In the third task the participant is required to explore one structural chemical formula through $\mu$ Graph and another one using ACD. The two formulae, aspirin and paracetamol (see Figures $3 \mathrm{c}$ and $3 \mathrm{~d}$ ), are presented in a counterbalanced way. At the end of each exploration, which can be conducted using all available exploration functions, participants are required to verbally describe the formula in the same way as in the first $\operatorname{task}^{2}$.

\section{RESULTS}

\section{First Task: Exploration}

For what concerns the exploration task, participants explored the formula for $4.8 \pm 0.42^{3}$ minutes. During this time, guided node exploration functionality was used $4.4 \pm 3.20$ times, while guided edges exploration was used $8.5 \pm 2.0$ times and free exploration was used $8.2 \pm 3.39$ times per participant. Node search by name function was invoked $2 \pm 1.49$ times per participant.

Out of 10 participants, 5 correctly reported all the atoms and bonds present. In 3 cases there was a single error in reporting bonds, and in 2 cases 1 bond and 1 atom were not reported. In all 9 cases atom positions were correctly reported. Instead, for bonds, errors over $15^{\circ}$ were 2 .

\footnotetext{
${ }^{2}$ Example transcript is available at: https://ewserver .di .unimi .it/mugraph/

${ }^{3}$ We use mean \pm standard deviation notation
}

One participant ran into difficulty to describe the correct position of one hydrogen atoms placed on the right side of the chemical formula, committing one atom and one bond positioning error. This participant was able to report all the other properties of the molecular structure. Another participant reported that the two atoms of carbon were not placed on the same line, committing one bond error.

It is remarkable that both the participants who committed these mistakes run the guided edges exploration more than once, but each time they stopped it before it completed. Hence, it is likely that they failed to get a proper mental image of the position of all the edges.

\section{Second Task: Editing}

For what concerns the second task, on average the participants took $4.7 \pm 0.67$ minutes in order to create the structural chemical formula. Only 2 of the participants placed all atoms and bonds. Instead, 3 participants omitted 2 atoms while 5 participants omitted one atom during the formula creation. Also, 4 participants omitted 2 bonds each and 4 omitted one bond each.

In terms of placement accuracy, 8 participants placed all inserted atoms within the threshold correctness range. Instead, two participant placed one hydrogen atom too far from the intended position, while all participants defined all bonds correctly.

All exploration functionalities were used for creating the structural chemical formula by each user, as well as to check the correctness of the structure created. Additionally, two deletion operations were performed in order to correct erroneously created atoms by two distinct participants.

\section{Third Task: Comparison with ACD}

In Task 3, participants required on average $5.3 \pm 0.82$ minutes to explore a structural chemical formula with $\mu \mathrm{Graph}$ and $4.4 \pm 0.70$ minutes with ACD. The difference is statistically significant based on analysis with a paired Student's t-test $(p<0.05)$.

In terms of errors, with $\mu$ Graph one participant forgot to mention one atom and one bond, and two other participants did not mention one atom and one bond respectively. Instead, considering ACD, two participants forgot two atoms each, and two others did not position one atom each. Most importantly, all participants misplaced at least one bond with ACD, two of them did not place two bonds, and other two did not place three bonds. The difference with respect to $\mu \mathrm{Graph}$ was found to be significant also in this case $(p<0.005)$.

This result shows that, while $\mu$ Graph interaction results to be slower due to the presence of a number of diverse exploration functionalities, it also provides a more accurate understanding of structural chemical formulae. Moreover, with $\mu$ Graph the participants were also able to describe the geometric properties of the explored molecule, namely the position of atoms and the angles between bonds, information which is not provided by ACD. 


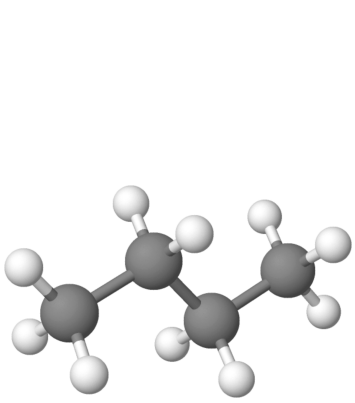

(a) Butane

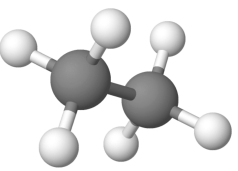

(b) Ethane

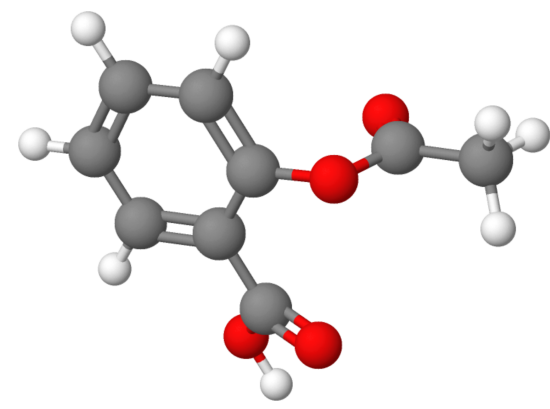

(c) Aspirin

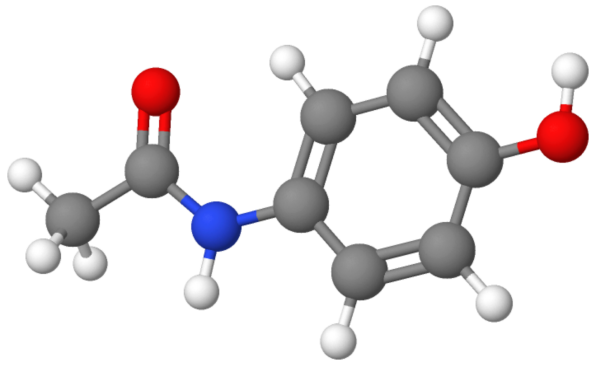

(d) Paracetamol

Figure 3: Structural chemical formulae used

From the open-ended questions two main remarks can be reported. First, 6 participants regarded the guided exploration modalities as useful and easy-to-use. Second, 4 participants reported that the hierarchical exploration available in ACD would also be useful in $\mu$ Graph and, vice versa, the search of node by name and the guided explorations would be useful in $\mathrm{ACD}$, too. This suggests that including hierarchical navigation of ACD into $\mu$ Graph could further improve it structural chemical formula exploration and creation capabilities.

\section{Limitations}

The results highlight three main limitations of $\mu \mathrm{Graph}$ :

\section{Working volume dimensions.}

Even though the current work volume enables users to place a number of nodes and edges sufficient to represent many structural chemical formulae, a wider work volume would enable both to represent more complex formulae and to avoid confusion in detecting too close nodes and intersecting edges.

\section{Partial Guided Exploration.}

Guided exploration was largely used and appreciated. However, when interrupted before all the graph elements were reached, the subjects committed errors in reporting the molecular structure. Hence, guided exploration will be modified to alert the user when nodes or edges have not yet been explored.

\section{Correct Placing of Elements.}

Correct placing of nodes and edges presents some difficulties. Thus, the mechanism for placing these elements will be enhanced by providing with further information about the elements close to the pointer.

\section{CONCLUSIONS AND FUTURE WORK}

This work presents $\mu \mathrm{Graph}$, a multimodal system to support people with VIB to create, edit and explore structural chemical formulae in a three-dimensional workspace using haptic and auditory feedback. The usability evaluation with ten participants with VIB has shown that the system is usable both for editing and exploring graphs. It is remarkable that all the system's features were used by the participants during the evaluation in order to complete editing and exploration tasks. In particular, guided exploration of nodes and edges proved to be the most frequently used system features. Actually, all participants used this exploration technique both in exploration tasks and in editing tasks.
The comparative evaluation between $\mu$ Graph and ACD, a system for exploring structural chemical formulae in chemistry through speech feedback, provided evidence that both systems are effective to understand structure of the graph, namely the structure of the nodes and connections between edges. Nonetheless, $\mu$ Graph provides a better understanding of the geometric structure of the graph, as well as the position of nodes and edges in the three-dimensional space, which is not provided in ACD.

Furthermore, the comparative evaluation suggests that both systems can be improved. In particular, $\mu$ Graph could be enhanced by including the hierarchical exploration paradigm available in ACD. On the other hand, the guided exploration techniques available in our solution could be used to improve the usability of a system that does not rely on haptic feedback like ACD.

The future work on $\mu$ Graph will be conducted along four main directions:

- The first improvement will consist in the implementation of the capability to import graph representations from standard formats adopted in the world of scientific publishing (e.g. CML for structural chemical formulae). The ability of the system to import from standard formats would provide access to blind and sight impaired people to a great deal educational resources.

- The second improvement will consist in the implementation and the integration of a hierarchical exploration modality in the system, as emerged from the comparative evaluation with ACD.

- The third direction will involve the design and development of further editing and exploration techniques, and personalization of the existing interaction to be suitable also for partially sighted people in order to extend the use of the system to a larger target population.

- The fourth research direction concerns the investigation of multimodal interaction paradigms relying on diverse haptic devices, including commodity devices, which would enable the usage of our technique for education in low-income settings. 


\section{REFERENCES}

[1] Dragan Ahmetovic, Tiziana Armano, Cristian Bernareggi, Michele Berra, Anna Capietto, Sandro Coriasco, Nadir Murru, Alice Ruighi, and Eugenia Taranto. 2018. Axessibility: a LaTeX Package for Mathematical Formulae Accessibility in PDF Documents. In International ACM SIGACCESS conference on Computers \& accessibility. ACM.

[2] Dragan Ahmetovic, Cristian Bernareggi, João Guerreiro, Sergio Mascetti, and Anna Capietto. 2019. AudioFunctions.web: Multimodal Exploration of Mathematical Function Graphs. In International Cross-Disciplinary Conference on Web Accessibility. ACM.

[3] Suzanne P Balik, Sean P Mealin, Matthias F Stallmann, Robert D Rodman, Michelle L Glatz, and Veronica J Sigler. 2014. Including blind people in computing through access to graphs. In International ACM SIGACCESS conference on Computers \& accessibility. ACM.

[4] Abeer S Bayousuf, Hend S Al-Khalifa, and AbdulMalik S Al-Salman. 2013. Towards the development of haptic-based interface for teaching visually impaired arabic handwriting. In International ACM SIGACCESS Conference on Computers and Accessibility. ACM.

[5] Cristian Bernareggi, Christian Comaschi, Giancarlo Dalto, Piero Mussio, and Loredana Parasiliti Provenza. 2008. Multimodal exploration and manipulation of graph structures. In International Conference on Computers for Handicapped Persons. Springer.

[6] Craig Brown and Amy Hurst. 2012. VizTouch: automatically generated tactile visualizations of coordinate spaces. In International Conference on Tangible, Embedded and Embodied Interaction. ACM.

[7] Morten Fjeld and Benedikt M Voegtli. 2002. Augmented chemistry: An interactive educational workbench. In International Symposium on Mixed and Augmented Reality. IEEE.

[8] Giovanni Fusco and Valerie S Morash. 2015. The tactile graphics helper: providing audio clarification for tactile graphics using machine vision. In International ACM SIGACCESS Conference on Computers \& Accessibility. ACM.

[9] John A. Gardner and Vladimir Bulatov. 2006. Scientific Diagrams Made Easy with IVEOTM. In International Conference on Computers Helping People with Special Needs. Springer.
[10] Morton A Heller and Edouard Gentaz. 2013. Psychology of touch and blindness. Psychology press.

[11] Michele Hu. 2015. Exploring new paradigms for accessible 3D printed graphs. In International ACM SIGACCESS Conference on Computers \& Accessibility. ACM.

[12] David K McGookin and Stephen A Brewster. 2006. MultiVis: improving access to visualisations for visually impaired people.

[13] Rameshsharma Ramloll, Wai Yu, Stephen Brewster, Beate Riedel, Mike Burton, and Gisela Dimigen. 2000. Constructing sonified haptic line graphs for the blind student: first steps.

[14] Michael J Robertson and William L Jorgensen. 2015. Illustrating concepts in physical organic chemistry with 3D printed orbitals. Journal of Chemical Education.

[15] Makoto Sato, Xiangning Liu, Jun Murayama, Katsuhito Akahane, and Masaharu Isshiki. 2008. A haptic virtual environment for molecular chemistry education. In Transactions on Edutainment I. Springer.

[16] Lei Shi, Idan Zelzer, Catherine Feng, and Shiri Azenkot. 2016. Tickers and talker: An accessible labeling toolkit for 3D printed models. In CHI Conference on Human Factors in Computing Systems. ACM.

[17] Alejandro Jarillo Silva, Omar A Domínguez Ramirez, Vicente Parra Vega, and Jesus P Ordaz Oliver. 2009. Phantom omni haptic device: Kinematic and manipulability. In Electronics, Robotics and Automotive Mechanics Conference. IEEE.

[18] Derrick W Smith and Sinikka M Smothers. 2012. The role and characteristics of tactile graphics in secondary mathematics and science textbooks in braille. Journal of Visual Impairment \& Blindness.

[19] Volker Sorge, Dragan Ahmetovic, Cristian Bernareggi, and John Gardner. 2019. Scientific Documents. In Web Accessibility. Springer.

[20] Volker Sorge, Mark Lee, and Sandy Wilkinson. 2015. End-to-end solution for accessible chemical diagrams. In Web for All Conference. ACM.

[21] Wai Yu and Stephen Brewster. 2003. Evaluation of multimodal graphs for blind people. Universal Access in the Information Society.

[22] John S Zelek, Sam Bromley, Daniel Asmar, and David Thompson. 2003. A haptic glove as a tactile-vision sensory substitution for wayfinding. Journal of Visual Impairment and Blindness. 